\title{
Comparación de psicopatologías y apoyo prestado a cuidadores de enfermos de Alzheimer por la farmacia comunitaria y las asociaciones de cuidadores
}

\section{Natalia Vérez-Cotelo ${ }^{1,6}$, Marcos Ríos-Lago ${ }^{2}$, N. Floro Andrés-Rodríguez ${ }^{3,6}$, José A. Fornos-Pérez ${ }^{4,6}$, J. Carlos Andrés-Iglesias ${ }^{5,6}$, Rocío Mera-Gallego ${ }^{5,6}$}

1. Doctora en Farmacia. Universidad San Pablo CEU. Escuela de Magisterio de Vigo. 2. Doctor en Psicología. Dpto. Psicología Básica II. Facultad de Psicología. Universidad Nacional de Educación a Distancia (UNED). 3. Doctor en Farmacia. Farmacéutico comunitario en Vigo (España). 4. Doctor en Farmacia. Farmacéutico comunitario en Cangas do Morrazo (España). 5. Farmacéutico comunitario en Vigo (España). 6. Grupo Berbés de Investigación y Docencia (www.grupoberbes.es).

\section{PALABRAS CLAVE}

Cuidador familiar, farmacia comunitaria, asociaciones de familiares, sobrecarga, psicopatologías, apoyo social

\section{ABREVIATURAS}

EA: enfermedad/ enfermo de Alzheimer CFEA: cuidador familiar de enfermo de Alzheimer

AFA: asociación de familiares de enfermos de Alzheimer IC: índice de confianza DE: desviación estándar

\section{RESUMEN}

Introducción: Los cuidadores familiares de enfermos de Alzheimer (CFEA) sufren sobrecarga y psicopatologías derivadas del cuidado. Objetivo: comparar el estado de sobrecarga, ansiedad, depresión y apoyo social percibido por los CFEA que pertenecen a una asociación de familiares de enfermos de Alzheimer (AFA) y los que no pertenecen y son atendidos en farmacia comunitaria.

Métodos: Estudio observacional transversal. 175 CFEA, divididos en dos grupos. Uno de 25 CFEA captado entre cuidadores que acuden regularmente a una farmacia y no pertenecían a ninguna asociación (FCIA). El segundo grupo (AFA) de 150 CFEA pertenecientes a 7 AFA de Galicia. Se registraron variables sociodemográficas y se administraron los cuestionarios: inventario de depresión de Beck, STAI-Cuestionario de ansiedad, escala de sobrecarga del cuidador de Zarit, escala Duke-UNC del apoyo social percibido.

Resultados: En los dos grupos el perfil de cuidador corresponde con una mujer de 56 años, familiar de primer grado del EA, con estudios primarios. Sin diferencias significativas entre grupos en edad $(p=0,931)$ ni nivel educativo $(p=0,508)$. Tampoco se encontraron diferencias en estado civil $(\mathrm{p}=0,468)$, ni en situación laboral $(\mathrm{p}=0,851)$. Diferencias significativas: ansiedad, sobrecarga y apoyo social. El grupo AFA obtuvo puntuaciones más altas en sobrecarga $(t=3,162 ; p=0,002)$, ansiedad $(t=2,054 ; p=0,046)$ y apoyo social percibido $(t=2,755 ; p=0,006)$. Sin diferencias significativas en depresión $(\mathrm{t}=-0,881 ; \mathrm{p}=0,380)$.

Conclusiones: Los cuidadores familiares del grupo AFA mostraron mayor sobrecarga y ansiedad. Los resultados de este trabajo respaldan la utilidad del farmacéutico comunitario en la detección de psicopatologías asociadas al cuidado del EA y la atención a los cuidadores.

El trabajo presentado en este artículo forma parte de la tesis doctoral de Natalia Vérez Cotelo, presentada en la UNED en enero de 2016 con la que obtuvo la calificación de Sobresaliente cum Laude y el Premio Cinfa 2015 a las mejores iniciativas de la Farmacia Española.

Se presentó como comunicación oral en el $5^{\text {th }}$ World Congress on Neurology and Therapeutics en Londres 2016 y en el XXVIII Congreso de Gerontología y Geriatría en Lugo 2016. Obtuvo el Premio a la mejor comunicación en el apartado de Educación para la Salud y Salud Pública en el Congreso Nacional de Farmacéuticos Comunitarios de SEFAC en Zaragoza, 2016.

Recibido: 12/7/2016

Aceptado: 30/8/2016

Disponible online: 1-9-2016
Financiación: Ninguna ajena.

Conflicto de intereses: Les autores declaran no existir conflicto de intereses en relación con el contenido del artículo. Contribución a la autoría: NVC: participó en el diseño del estudio, el desarrollo de la investigación, el análisis de los resultados y su tratamiento estadístico, en la redacción del manuscrito y la revisión del contenido final. MRL: participó en el diseño del estudio, el análisis de los resultados y su tratamiento estadistico y la revisión del contenido final. NFAR: participó en el diseño del estudio, el análisis de los resultados y su tratamiento estadistico, en la redacción del manuscrito y la revisión del contenido final. JAFP: participó en el diseño del estudio, el análisis de los resultados y su tratamiento estadístico y en la revisión del contenido final del manuscrito. JCAl: participó en el diseño del estudio, el análisis de los resultados y su tratamiento estadistico y en la revisión del contenido final del manuscrito. RMG: participó en el desarrollo de la investigación, el análisis de los resultados y en la revisión del contenido final del manuscrito.

Cite este artículo como: Vérez-Cotelo N, Ríos-Lago M, Andrés-Rodriguez NF, Fornos-Pérez JA, Andrés-Iglesias JC, Mera-Gallego R. Comparación de psicopatologias y apoyo prestado a cuidadores de enfermos de Alzheimer por la farmacia comunitaria y las asociaciones de cuidadores. Farmacéuticos Comunitarios. 2016 Sep 30; 8(3):5-11. doi:10.5672/FC.2173-9218.(2016/Vol8).003.02 Autora para correspondencia: Natalia Vérez-Cotelo (nataliavc@grupoberbes.es).

ISSN 1885-8619 @SEFAC (Sociedad Española de Farmacia Familiar y Comunitaria). Todos los derechos reservados. 
Comparison of psychopathologies and support given to caregivers of Alzheimer patients by the community pharmacy and caregiver associations

\section{KEYWORDS}

Family caregiver, community pharmacy, family associations, burden, psychopathologies, social support

\section{ABSTRACT}

Introduction: Family caregivers of Alzheimer patients (FCAP) suffer from excessive burdens and psychopathologies deriving from the care. Objective: compare the level of excessive burdens, anxiety, depression and social support perceived by FCAPs who belong to a family members of Alzheimer patients association (FAA) and those who don't and are attended to at the community pharmacy.

Methods: Observational transversal study. 175 FCAPs, divided into two groups. One with 25 FCAPs formed by caregivers who go regularly to a pharmacy and don't belong to an association (FAA). The second group (FAA) of 150 FCAPs belonging to 7 FAAs in Galicia. Sociodemographic variables were recorded and questionnaires were handed out: Beck Depression Inventory, STAI-Anxiety questionnaire, Zarit caregiver burden scale, Duke-UNC scale of perceived social support.

Results: In both groups the profile of the caregiver is a 56 year old woman, direct family member of the Alzheimer's patient with primary education. There are no significant differences between the groups in age $(p=0.931)$ or educational level $(p=0.508)$. Nor were there differences in marital status $(p=0.468)$ or employment status ( $p=0.851)$. Significant differences: anxiety, burden and social support. The FAA had higher scores in burden ( $t=3.162 ; \mathrm{p}=0.002)$, anxiety $(t=2.054 ; p=0.046)$ and perceived social support $(t=2.755 ; p=0.006)$. There were no significant differences in depression ( $t=0.881 ; \mathrm{p}=0.380)$.

Conclusions: Family caregivers of the FAA group displayed higher levels of burden and anxiety. The results of this study support the use of community pharmacies for detecting psychopathologies associated with caring for Alzheimer's patients and healthcare for caregivers.

\section{Introducción}

La enfermedad de Alzheimer (EA) es una enfermedad neurodegenerativa caracterizada por un deterioro progresivo en las áreas cognitiva, funcional, conductual/psicológica y global [1,2]. La evolución de la enfermedad varía de unos pacientes a otros, con un inicio lento pero con una progresión implacable pues, a medida que evoluciona van apareciendo distintos síntomas que hacen que los enfermos vayan empeorando su situación y cada vez necesiten más ayuda de otras personas [1,2,3]. La supervivencia media actual tras el diagnóstico es de entre 8 y 14 años y no suele prolongarse más allá de 15 años [4].

En España no existen estudios epidemiológicos actualizados, sin embargo se estima que actualmente hay entre 500.000 y 800.000 pacientes con esta patología, incluyendo los casos no diagnosticados. En Galicia se calculan en 70.000 [5].

Las características de la enfermedad de Alzheimer hacen que los enfermos necesiten ayuda de otras personas para desenvolverse, ayuda que es prestada a través del cuidado informal o del cuidado formal. El cuidador informal se corresponde con un familiar o una persona cercana que le presta apoyo desinteresado y voluntario (no remunerado) y que además no suele poseer capacitación [6].
Los cuidadores familiares de los enfermos de Alzheimer (CFEA) viven junto al enfermo a lo largo de todas las fases de la enfermedad y sufren en primera persona el deterioro progresivo e irreversible de su familiar, lo cual tiene un considerable coste en su propio bienestar [3,7]. Esta situación puede implicar la aparición de determinados síntomas que condicionarán el adecuado funcionamiento de los familiares como cuidadores $[3,8,10]$.

El impacto sobre la salud del cuidador se manifiesta a nivel físico: cefaleas tensionales, astenia o fatiga crónica, alteración del ciclo sueño-vigilia e insomnio, problemas osteoarticulares, dolores musculares, lumbalgias, artritis, problemas gastrointestinales, obesidad, diabetes, hipertensión, problemas cardiovasculares [7,11]. Y psíquico: estrés, sobrecarga, inseguridad en el cuidado del enfermo, sensación de aislamiento social, manifestaciones psicosomáticas de ansiedad y/o depresión y en fases sucesivas se puede intensificar el cansancio psicofísico y miedo a la pérdida del ser querido $[8,12,13]$.

Además, los CFEA se enfrentan a una serie de consecuencias sociales, afectando de forma notable al trabajo, la economía, las relaciones familiares y sociales o el uso del tiempo y del ocio $[9,14,15]$.

Las asociaciones de familiares de enfermos de Alzheimer (AFA) desarro- llan actividades y recursos dirigidos a los cuidadores y las familias. Incluyen información sobre la enfermedad, el manejo del paciente en las distintas fases, recursos existentes en la Administración, utilización de servicios de asistencia domiciliaria, unidades de respiro familiar o servicios de voluntariado, apoyo psicológico individual o en grupo y asesoramiento legal. Las AFA realizan también actividades dirigidas a la sociedad en general, como concienciar sobre el impacto de la enfermedad, promocionar medidas de prevención, promocionar la investigación, colaborar con otras entidades y actuar de interlocutor con las administraciones públicas [5].

No hay publicaciones basadas en la evidencia que avalen los beneficios de pertenecer a una AFA, pero hay un cierto consenso entre los expertos en recomendar este tipo de recurso ya que produce un elevado grado de satisfacción en las familias y los cuidadores que lo utilizan [16].

A partir del momento en que el médico especialista establece un diagnóstico de enfermedad de Alzheimer y el tratamiento correspondiente, es importante que se mantenga una buena relación entre el enfermo (en las primeras fases de la enfermedad), su cuidador, la familia, el médico de familia y el farmacéutico comunitario. Una buena comunicación farmacéutico-cuidador redundará en una 
mejora de la adherencia al tratamiento de los pacientes con enfermedad de Alzheimer. La farmacia comunitaria es una puerta de entrada al sistema sanitario, cercana y accesible, en la que se establece un clima de confianza entre farmacéutico, paciente y cuidador que favorece la comunicación $[17,18]$. Este clima de confianza facilita el acceso a cuidadores no demandantes de ayuda, que la rechazan o dejan de utilizarla, por falta de percepción de necesitarla, escasa disponibilidad de tiempo, imposibilidad de dejar al mayor con alguien o presentar problemas de salud como han constatado algunos autores [9].

En el presente estudio se pretende comparar el estado de sobrecarga, ansiedad, depresión y apoyo social percibido por los cuidadores familiares de enfermos de Alzheimer que pertenecen a asociaciones de familiares y los que no pertenecen a ninguna asociación y son atendidos en farmacia comunitaria.

\section{Métodos \\ Diseño}

Estudio observacional transversal desarrollado entre julio de 2014 y julio de 2015.

\section{Sujetos}

Participaron en el estudio cuidadores familiares de enfermos de Alzheimer, divididos en dos grupos: uno formado por 25 cuidadores que acudían regularmente a una farmacia comunitaria de la ciudad de Pontevedra (Galicia) y no pertenecían a ninguna asociación (grupo FCIA) y un segundo grupo (grupo AFA) de CFEA pertenecientes a AFA, para lo que se contactó con siete AFA de Galicia, ofreciendo a sus asociados la participación en el estudio. Se obtuvieron 150 respuestas válidas de CFEA que constituyeron el grupo AFA.

Criterios de inclusión: cuidadores principales informales, mayores de 18 años capaces de leer y entender los instrumentos de medida. Con vínculo de parentesco con el EA, que convivían con él y que firmaron el consentimiento informado.

Se ofreció participar en el estudio a todos los CFEA que acudian a la farmacia a retirar medicamentos para familiares diagnosticados de Alzheimer.
Variables e instrumentos

de medida

En una hoja de registro diseñada ad hoc se recogieron las variables sociodemográficas y relacionadas con el cuidado: sexo, edad, estado civil, nivel de estudios, situación laboral, parentesco con el EA, si el CFEA recibía ayuda para el cuidado del enfermo por parte de algún familiar o servicio social o privado de ayuda en domicilio. Escala Duke-UNC [19] de valoración del apoyo social. Variables clínicas: sobrecarga (escala de sobrecarga del cuidador de Zarit) [20], síntomas de ansiedad (STAI-Cuestionario de ansiedad-estado) [21], síntomas de depresión (inventario de depresión de Beck) [22].

\section{Cálculo del tamaño muestral}

Para el cálculo del tamaño muestral necesario se tuvo en cuenta los resultados de un trabajo anterior, en el que se realizó un pilotaje previo al presente estudio [23], resultando una puntuación media del test de Zarit de 25,0 $(D E=8,3)$. Para una precisión de 1,5 unidades en la estimación de una media mediante un intervalo de confianza (IC) de 95\%, y teniendo en cuenta una hipótesis bilateral, la muestra requerida resultó de al menos 118 unidades experimentales.

\section{Tratamiento estadístico}

Para el tratamiento estadístico se usó el programa estadístico SPSS $^{\circledR}$ 15.0 para Windows ${ }^{\circledR}$. Las variables cualitativas se expresaron como porcentajes y las variables cuantitativas como media (desviación estándar). Los límites de confianza se calcularon al 95\% (IC), utilizando los test de chi-cuadrado para variables cualitativas y $\mathrm{t}$ de Student para variables cuantitativas. Se utilizaron técnicas analíticas de correlación de Pearson. La significación estadística se fijó en $\mathrm{p}<0,05$.

Este estudio fue aprobado por el Comité Ético de Investigación Clínica de la Comunidad Autónoma de Galicia y obtuvo el aval científico de la Sociedade Galega de Xerontoloxía e Xeriatría, del Colegio Oficial de Farmacéuticos de Pontevedra y la Federación de Asociacións Galegas de Familiares de Enfermos de Alzheimer e Outras Demencias.

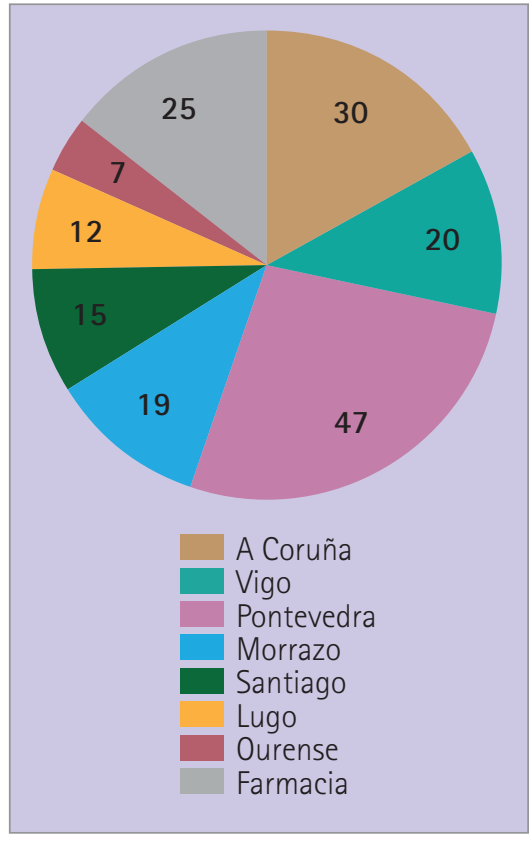

Figura 1 Procedencia de los cuidadores que componen la muestra

\section{Resultados}

La muestra estuvo constituida por 175 CFEA, 135 (77,1\%) mujeres y $40(22,9 \%)$ hombres. Su procedencia (AFA y FCIA) se muestra en la figura 1.

La edad media fue de 56,3 años ( $\mathrm{DE}=11,9 ; 22-87)$. Las características demográficas y las relacionadas con el cuidado se presentan en la tabla 1 . No se encontraron diferencias significativas entre grupos en edad $(p=0,931)$, estado civil $(p=0,468)$, grado de parentesco con el EA ( $p=0,566)$, nivel de estudios $(p=0,508)$ ni situación laboral $(p=0,851)$. Sí en cuanto al disfrute de ayuda $(p=0,010)$ y al apoyo social percibido $(\mathrm{p}=0,006)$, ambos mayores en el grupo AFA.

La puntuación media del inventario de depresión de Beck fue en el grupo AFA de 11,4 ( $\mathrm{DE}=8,6 ; 0-40)$ y en el grupo FCIA de 13,1 (DE=10,6; 0-37), diferencia no significativa $(\mathrm{t}=-0,881$; $p=0,380$ ). La media del cuestionario Stai-estado de ansiedad resultó de $27,9(\mathrm{DE}=12,0 ; 3-60)$ en el grupo AFA $y$ de $23,9(D E=8,3 ; 10-40)$ en el grupo FCIA, diferencia significativa $(t=2,054$; $\mathrm{p}=0,046)$. En la valoración de la sobrecarga el resultado medio del grupo AFA fue de 57,8 (DE=14,6; 27-101) y en el grupo FCIA de 47,2 ( $\mathrm{DE}=13,5$; 20-80), diferencia que también resultó significativa $(t=3,162 ; p=0,002)$.

Las variables clínicas: síntomas de depresión, ansiedad y nivel de sobrecarga, distribuidas según las puntuaciones 
Tabla 1 Variables sociodemográficas y relacionadas con el cuidado

\begin{tabular}{|c|c|c|c|c|}
\hline & Grupo AFA n=150 & Grupo FCIA $n=25$ & & \\
\hline Edad m (DE) Rango & $56,3(11,9) 22-87$ & $56,6(12,8) 39-87$ & $t=-0,087$ & $p=0,931$ \\
\hline $\begin{array}{l}\text { Estado civil n (\%) } \\
\text { Soltero } \\
\text { Casado } \\
\text { Viudo } \\
\text { Divorciado o separado }\end{array}$ & $\begin{array}{c}21(14,0) \\
115(76,7) \\
5(3,3) \\
9(6,0)\end{array}$ & $\begin{array}{c}2(8) \\
21(84) \\
0(0) \\
2(8)\end{array}$ & $\chi^{2}=2,542$ & 0,468 \\
\hline $\begin{array}{l}\text { Parentesco n (\%) } \\
\text { Hija } \\
\text { Hijo } \\
\text { Esposa } \\
\text { Esposo } \\
\text { Nuera } \\
\text { Sobrina } \\
\text { Nieta }\end{array}$ & $\begin{array}{c}77(51,4) \\
21(14,0) \\
26(17,3) \\
14(9,3) \\
7(4,7) \\
3(2,0) \\
2(1,3)\end{array}$ & $\begin{array}{c}15(60) \\
1(4) \\
3(12,0) \\
4(16,0) \\
1(4,0) \\
1(4,0) \\
0(0,0)\end{array}$ & $\chi^{2}=4,832$ & 0,566 \\
\hline $\begin{array}{l}\text { Nivel de estudios n (\%) } \\
\text { Estudios primarios } \\
\text { Secundaria } \\
\text { Bachillerato/FP } \\
\text { Universitarios }\end{array}$ & $\begin{array}{l}53(35,3) \\
27(18,0) \\
22(14,7) \\
48(32,0)\end{array}$ & $\begin{array}{l}12(48) \\
3(12) \\
8(32) \\
2(8)\end{array}$ & $\chi^{2}=0,664$ & 0,508 \\
\hline $\begin{array}{l}\text { Trabajo remunerado } \mathrm{n}(\%) \\
\text { Sí } \\
\text { No }\end{array}$ & $\begin{array}{l}62(41,3) \\
88(58,7)\end{array}$ & $\begin{array}{l}10(40,0) \\
15(60,0)\end{array}$ & $\chi^{2}=0,035$ & 0,851 \\
\hline $\begin{array}{l}\text { Recibe ayuda n (\%) } \\
\text { Sí } \\
\text { No }\end{array}$ & $\begin{array}{l}95(63,3) \\
55(36,7)\end{array}$ & $\begin{array}{c}9(36,0) \\
16(64,0)\end{array}$ & $\chi^{2}=6,640$ & 0,010 \\
\hline Apoyo social m (DE) Rango & $39,5(11,1) 12-55$ & $32,6(13,3) 12-53$ & $t=2,755$ & 0,006 \\
\hline
\end{tabular}

Tabla 2 Variables clínicas

\begin{tabular}{|l|c|c|}
\hline & $\begin{array}{c}\text { Grupo AFA } \\
n(\%)\end{array}$ & $\begin{array}{c}\text { Grupo FCIA } \\
n(\%)\end{array}$ \\
\hline $\begin{array}{l}\text { Depresión } \\
\text { Extrema }\end{array}$ & $0(0,0)$ & $0(0,0)$ \\
Severa & $7(4,7)$ & $3(12,0)$ \\
Moderada & $12(8)$ & $2(8,0)$ \\
Borderline & $14(9,3)$ & $3(12,0)$ \\
Ligero trastorno emocional & $26(17,3)$ & $3(12,0)$ \\
Normal & $91(60,7)$ & $14(56,0)$ \\
& $150(100,0)$ & $25(100,0)$ \\
\hline Ansiedad & & $4(16,0)$ \\
Mayor del percentil 75 & $61(40,6)$ & $21(84,0)$ \\
Menor del percentil 75 & $89(59,4)$ & $25(100,0)$ \\
& $150(100,0)$ & \\
\hline Sobrecarga & & $6(24,0)$ \\
Sobrecarga intensa & $79(52,6)$ & $7(28,0)$ \\
Sobrecarga leve & $33(22)$ & $12(48,0)$ \\
No sobrecarga & $38(25,4)$ & $25(100,0)$ \\
& $150(100,0)$ & \\
\hline
\end{tabular}

de los correspondientes cuestionarios se presentan en la tabla 2.

En las figuras 2 a 5 se presentan los niveles de depresión, ansiedad, sobrecarga y apoyo social percibido encontrados en los CFEA de cada una de las asociaciones participantes en el estudio y en el grupo FCIA. No se hallaron diferencias significativas entre ellas en las variables: depresión ( $t=1,657 ; p=0,136)$, ansiedad $(t=1,612 ; p=0,148)$ y sobrecarga $(t=0,865 ; p=0,522)$; sí en cuanto al apoyo social percibido $(t=2,856$; $\mathrm{p}=0,012$ ).

\section{Discusión}

El perfil sociodemográfico del cuidador principal familiar del enfermo de Alzheimer en Galicia coincide con el perfil del cuidador principal familiar de EA que existe en la literatura, tanto en estudios nacionales como internacionales. 


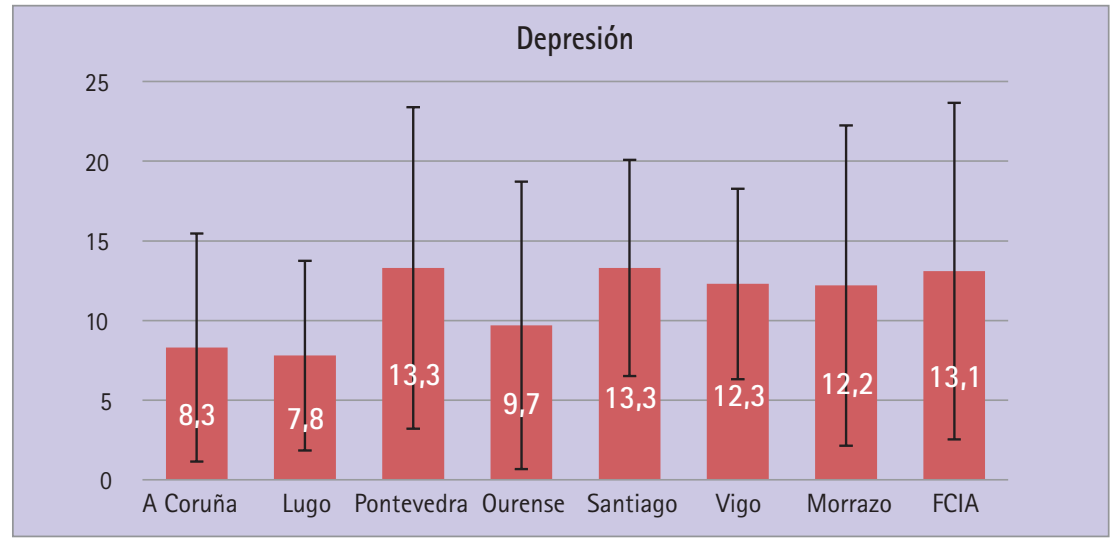

Figura 2 Resultados en depresión de las AFA participantes en el estudio y el grupo FCIA

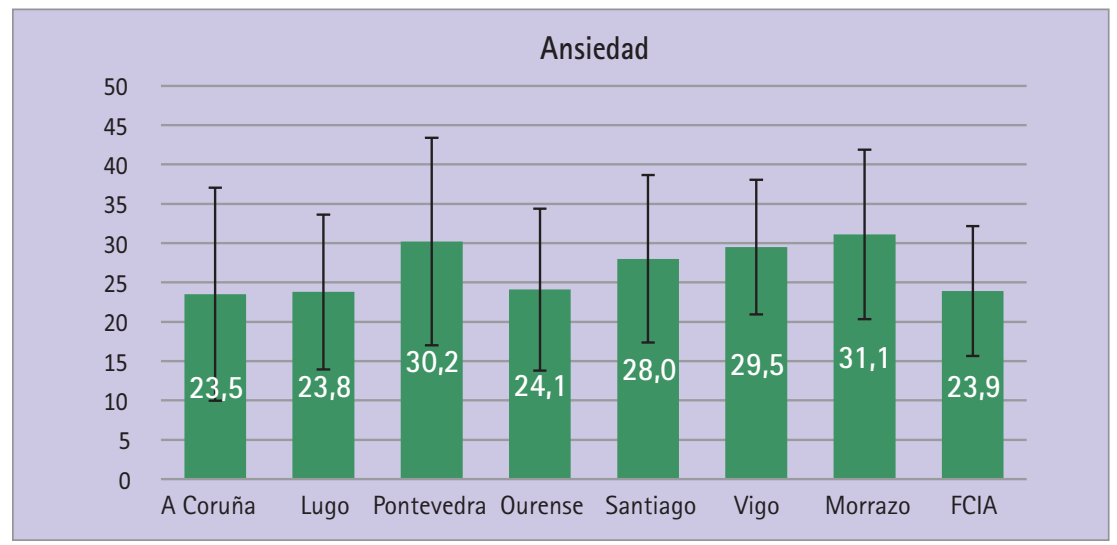

Figura 3 Resultados en ansiedad de las AFA participantes en el estudio y el grupo FCIA

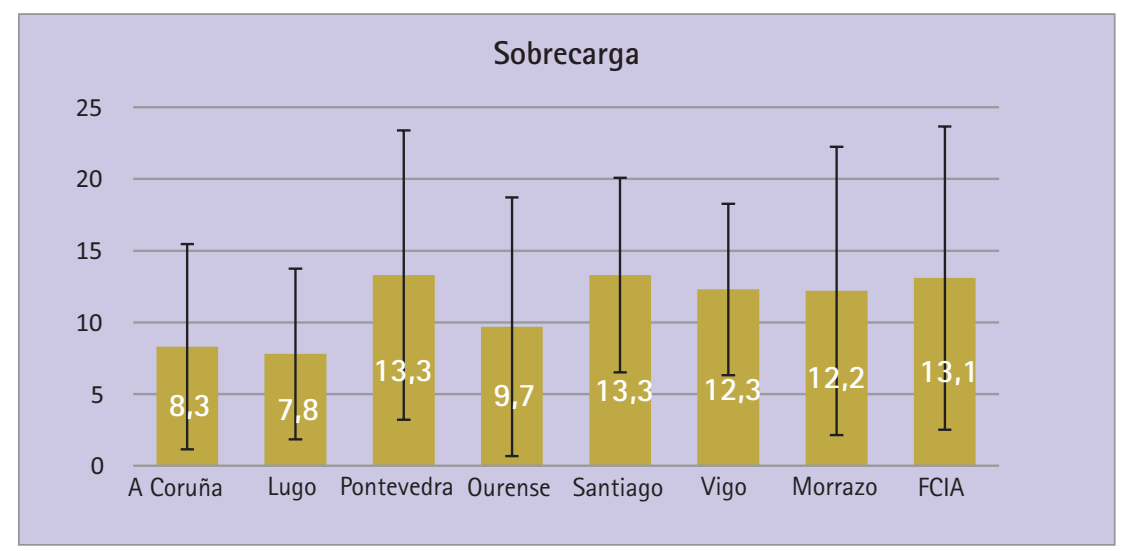

Figura 4 Resultados en sobrecarga de las AFA participantes en el estudio y el grupo FCIA

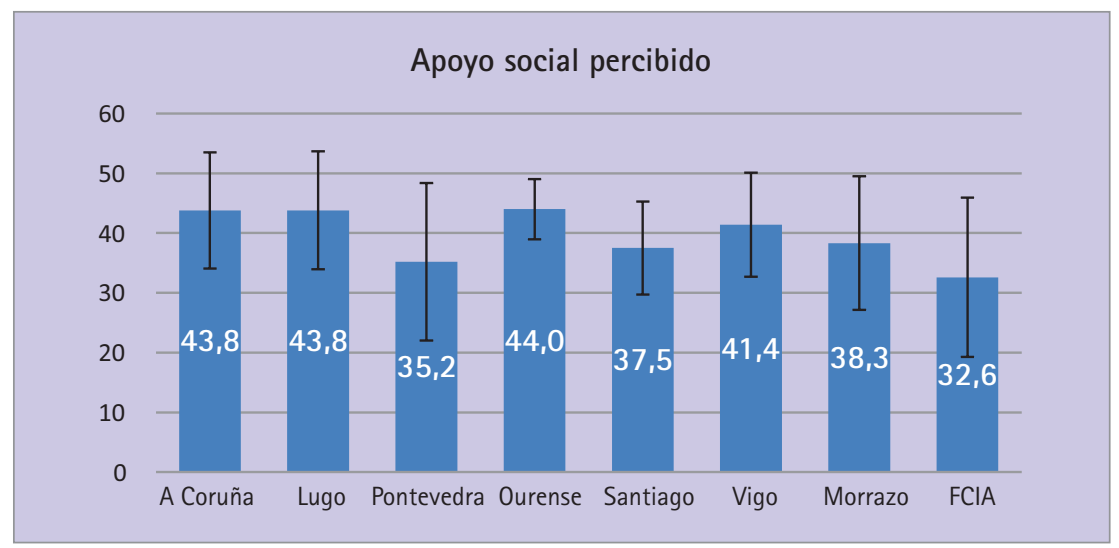

Figura 5 Resultados en apoyo social percibido de las AFA participantes en el estudio y el grupo FCIA
Éste parece ser independiente de las características culturales o sociales de la población estudiada y corresponde mayoritariamente a mujeres de mediana edad, de entre 40 y 60 años, familiares de primer grado del paciente (principalmente hijas), con estudios primarios, casadas y que no trabajan fuera del hogar [24-28].

Entre los CFEA participantes en nuestra investigación hemos encontrado un elevado porcentaje de cuidadores con estudios universitarios, algo que también hallaron otros autores [29]. La razón podría estar en que la aceptación a participar en el estudio y responder a la encuesta podría haberse producido por parte de cuidadores con elevado nivel educativo, más concienciados y participativos.

Los cuidadores pertenecientes a las AFA recibían más ayuda $(63,3 \%$ vs. $36,0 \%$ ) y en consecuencia percibían un mayor apoyo social, lo que coincide con otros estudios realizados en el contexto de AFA [26,30], que mostraron que los CFEA que pertenecen a AFA e instituciones relacionadas con los EA y sus cuidadores reciben una mayor ayuda para asistir al enfermo tanto por parte de otros familiares como por parte de las propias asociaciones.

En relación a las características clínicas, se han obtenido algunos resultados llamativos, como el hecho de que en el grupo AFA había un mayor porcentaje de CFEA con ansiedad y sobrecarga que en el grupo FCIA. La elevada carga obtenida en el grupo AFA también ha sido observada en otros estudios con muestras obtenidas en asociaciones de cuidadores [26,30,31] y podría explicarse por el hecho de que los cuidadores más sobrecargados son los que buscan ayuda en los recursos formales [32]. Diversas investigaciones han relacionado los síntomas conductuales y psicológicos asociados a la enfermedad de Alzheimer (SCPD) con el aumento de los niveles de sobrecarga de los cuidadores y para disminuir la frecuencia o intensidad de los síntomas cognitivos y psicológicos de la demencia es necesario recurrir a ayuda profesional [12,13]. Otra posible explicación podría ser que el grupo AFA se obtuvo tras una convocatoria hecha por las AFA de Galicia y quizá se podrían haber presentado los CFEA más sobrecargados y ansiosos mientras que en el grupo FCIA los CFEA 
reclutados para el estudio constituían la totalidad de los que recogían en la farmacia medicamentos para familiares diagnosticados de EA.

La participación voluntaria en el grupo AFA puede dar lugar a un sesgo de la muestra hacia los cuidadores más colaboradores, concienciados 0 que tuvieran ya la percepción de estar afectados por problemas de salud, como señalan algunos autores [33]. Además, algunos autores refieren un tipo de ansiedad anticipatoria en los cuidadores que pertenecen a AFA debida al "exceso" de información sobre cómo va a progresar la enfermedad y sus consecuencias y ver a otros EA en peor estado que su familiar [34]. En este sentido, habría que valorar la idoneidad de la información se transmite a las familias que llegan a un AFA porque se puede estar generando un problema de ansiedad por la anticipación de lo que puede venir. Además, los programas de transmisión de información a las familias deben modular ésta en función de la fase de evolución de la enfermedad en la que se encuentre el paciente. La menor proporción de CFEA con síntomas de sobrecarga y ansiedad encontrados en el grupo FCIA también podría explicarse porque en la farmacia comunitaria los CFEA reciben ayuda en cuanto a atención farmacéutica a su familiar: uso y administración de los medicamentos, sistemas personalizados de dosificación (SPD), consejo farmacéutico e información. Pero además, entre los servicios asistenciales que el farmacéutico comunitario presta a los cuidadores se encuentra la dispensación de medicamentos de indicación farmacéutica y a base de plantas medicinales para mejorar su estado de ansiedad e insomnio y también vitaminas o suplementos para mejorar su estado general, en mayor proporción entre los que pertenecían al grupo FCIA, lo que, en conjunto, puede haber contribuido también a mejorar los resultados obtenidos en este grupo [35].

Estos resultados, que no se han podido contrastar por la falta de estudios similares en nuestro medio, apuntan a un notable papel del farmacéutico comunitario y de la farmacia comunitaria como espacio de salud accesible y próximo, y abren el camino a posibles investigaciones futuras que determinen cómo la farmacia comunitaria puede ayudar a los CFEA.

\section{Conclusiones}

En los dos grupos el perfil de cuidador corresponde con una mujer de 56 años, familiar de primer grado del EA, con estudios primarios y que no trabaja fuera de casa.

Los CFEA de las AFA y los evaluados en la farmacia muestran un perfil clínico diferenciado en cuanto a los niveles de ansiedad y sobrecarga mostrados. Los cuidadores familiares que acuden a AFA, mostraron mayor sobrecarga y sintomatología ansiosa. Este hecho obligaría a reflexionar sobre el papel de las AFA en el cuidado del CFEA y qué intervenciones son más efectivas, lo que permitiría establecer protocolos de actuación unificados.

Los resultados de este trabajo respaldan la utilidad del farmacéutico comunitario en esa labor de detección de psicopatologías asociadas al cuidado del EA y la conveniencia de su inclusión en equipos multidisciplinares cuyo fin sea la reducción del malestar de los cuidadores.

\section{Referencias bibliográficas}

1. Ballard C, Gauthier S, Corbett A, Brayne C, Aarsland D, Jones E. Alzheimer's disease. Lancet. 2011; 377:1019-1031. doi:10.1016/S01406736(10)61349-9

2. Reitz C, Brayne C, Mayeux R. Epidemiology of Alzheimer disease. Nature Reviews Neurology. 2011; 7(3):137152. doi:10.1038/nrneurol.2011.2

3. Medrano M, Rosario RL, Payano AN, Capellán NR. Burden, anxiety and depression in caregivers of Alzheimer patients in the Dominican Republic. Dementia \&t Neuropsychologia. 2014; 8(4):384-388. doi:10.1590/S198057642014DN84000013

4. Peña Casanova J. Neurología de la conducta y neuropsicología. Madrid: Médica Panamericana; 2007.

5. Federación de Asociaciones Gallegas de Familiares de Enfermos de Alzheimer y otras Demencias. Dossier 2015. Santiago de Compostela: FAGAL; 2015.

6. Pereda C, De la Prada MA, Actis W, Rodríguez P, Sancho T. Cuidados en la Vejez. El apoyo informal. Madrid: Ministerio de Trabajo y Asuntos Sociales; 1999.

7. Laks J, Goren A, Dueñas H, Novick D, Kahle-Wrobleski K. Caregiving for patients with Alzheimer's disease or dementia and its association with psychiatric and clinical comorbidities and other health outcomes in Brazil. Int J Geriatr Psychiatry. 2016 Feb;
31(2):176-85. doi: 10.1002/gps.4309. Epub 2015 May 25. doi:10.1002/ gps.4309

8. Schulz R, Beach SR, Cook TB, Martire LM, Tomlinson JM, Monin JK. Predictors and consequences of perceived lack of choice in becoming an informal caregiver. Aging \&t Mental Health. 2012; 16(6):712-721. doi:10.1080/ 13607863.2011.651439

9. Martínez-Lage Álvarez P, Molinero Crespo AM, Tuneu Valls L. kNOW Alzheimer. Manual de Consulta para Farmacéuticos. Barcelona: Profármaco.2; 2014.

10. Peña-Longobardo LM, Oliva-Moreno J. Caregiver burden in Alzheimer's disease patients in Spain. Journal of Alzheimer's Disease. 2015; 43(4):12931302.

11. Manso ME, Sánchez MP, Cuéllar I. Salud y sobrecarga percibida en personas cuidadoras familiares de una zona rural. Clínica y Salud. 2013; 24(1):37-45. doi:10.5093/cl2013a5

12. Molinuevo JL, y Hernandez B. Profile of the informal career associated with the clinical management of the Alzheimer's disease patient refractory to symptomatic treatment of the disease. Neurología (English Edition). 2011; 26(9):518-527. doi:10.1016/j. nrleng.2011.05.001

13. Lou Q, Liu S, Huo YR, Liu M, Liu S, Ji Y. Comprehensive analysis of patient and caregiver predictors for caregiver burden, anxiety and depression in $\mathrm{Al}-$ zheimer's disease. Journal of Clinical Nursing. 2015; 24(17-18): 2668-2678. doi:10.1111/jocn.12870

14. Prieto C, Eimil M, López de Silanes C, Llanero M. [Internet]. Impacto social de la enfermedad de Alzheimer y otras demencias. 2011. Madrid: Fundación del Cerebro. [Acceso 6/6/2016]. Disponible en: http://www.fundaciondelcerebro. es/docs/imp_social_alzheimer.pdf

15. Losada A, Márquez M, Romero R, López J, Fernández-Fernández V, Nogales-González C. Atendiendo a las variadas problemáticas de los cuidadores familiares de personas con demencia: aportaciones de la terapia cognitivo-conductual y de la terapia de aceptación y compromiso. Clínica y Salud. 2015; 26:41-48. doi:10.1016/j. clysa.2015.02.001

16. Villars H, Oustric S, Andrieu S, Baeyens JP, Bernabei R, Brodaty $\mathrm{H}$, et al. The primary care physician and Alzheimer's disease: an international position paper. The Journal of Nutrition, Health \&t Aging. 2010; 14(2):110120. doi:10.1007/s12603-010-0022-0

17. Caelles N, Ibáñez J, Machuca M, Martínez-Romero F, Faus MJ. Entrevista farmacéutico-paciente en el programa Dáder de seguimiento farmacoterapéutico. Pharm Care Esp. 2002; 4:55-59. 
18. García-Delgado A, Machuca M, Murillo MD, de la Matta MJ, Martín MJ. Percepción de la labor asistencial del farmacéutico comunitario por los pacientes incluidos en un programa de seguimiento farmacoterapéutico. Seguim Farmacoter. 2003; 1(2):38-42.

19. De la Revilla L, Bailón E, Luna J, Delgado A, Prados MA, Fleitas L. Validación de una escala de apoyo social funcional para su uso en la consulta del médico de familia. Aten Primaria. 1991; 8:688-92.

20. Martín M, Salvadó I, Nadal S, Miji LC, Rico JM, Lanz y Taussing MI. Adaptación para nuestro medio de la escala de sobrecarga del cuidador (Caregiver Burden Interview) de Zarit. Revista de Gerontología. 1996; 6:338-346.

21. Spielberger CD, Gorsuch RL, Lushene RE. STAI. Inventario de Ansiedad Estado-Rasgo (versión española). (4a Ed.). Madrid: TEA; 1997.

22. Vázquez C, Sanz J. Fiabilidad y validez de la versión española del inventario para la depresión de Beck de 1978 en pacientes con trastornos psicológicos. Clínica y Salud. 1998; 10:59-81.

23. Vérez Cotelo N, Andrés Rodríguez NF, Fornos Pérez JA, Andrés Iglesias JC, Ríos Lago M. Burden and associated pathologies in family caregivers of Alzheimer's disease patients in Spain. Pharmacy Practice. 2015 AprJun;13(2): 521. doi:10.18549/PharmPract.2015.02.521

24. Badia X, Lara N, Roset M. Calidad de vida, tiempo de dedicación y carga percibida por el cuidador principal informal del enfermo de Alzheimer. Aten Primaria. 2004; 34:170-177. doi:10.1016/S0212-6567(04)78904-0

25. Conde JL. La familia y los cuidados informales en la demencia. Diferencias entre cónyuges e hijos cuidadores. En Alberca R, López-Pousa S [Eds.]. Enfermedad de Alzheimer y otras Demencias (pp. 101-11). 4a Ed. Madrid: Panamericana; 2010.

26. Turró 0, López-Pousa S, Vilalta J, Turón A, Pericot I, Lozano M, et al. Valor económico anual de la asistencia informal de la Enfermedad de Alzheimer. Rev Neurol. 2010; 51(4): 201-207.

27. Manso, M.E., Sánchez, M.P. y Cuéllar, I. Salud y sobrecarga percibida en personas cuidadoras familiares de una zona rural. Clínica y Salud. 2013; 24(1):37-45. doi:10.5093/cl2013a5

28. Reed C, Belger M, Dell'Agnello G, Wimo A, Argimon JM, Bruno G, et al. Caregiver burden in Alzheimer's disease: Differential associations in adult-child and spousal caregivers in the GERAS observational study. Dement Geriatr Cogn Dis Extra. 2014; 4(1):51-64. doi:10.1159/000358234

29. Papastavrou E, Andreou P, Middleton N, Papacostas S, Georgiou I. Factors associated with quality of life among family members of patients with dementia in Cyprus. International Psychogeriatrics. 2014; 26(3):443-452. doi:10.1017/S104161021300224X

30. Fernández-Lansac V, Crespo López M, Cáceres R, Rodríguez-Poyo M. Resiliencia en cuidadores de personas con demencia: estudio preliminar. Revista
Española de Geriatría y Gerontología. 2012; 47(3):102-109. doi:10.1016/j. regg.2011.11.004

31. Abdollahpour I, Noroozian M, Nedjat S, Majdzadeh R. Caregiver burden and its determinants among the family members of patients with dementia in Iran. Int J Prev Med; 2012; 3(8):544.

32. Artaso B, Goñi A, Biurrun A. Cuidados informales en la demencia: predicción de sobrecarga en cuidadoras familiares. Revista Española de Geriatría y Gerontología. 2003; 38(4):212-218. doi:10.1016/S0211-139X(03)74886-0

33. Pinquart M, Sörensen S. Correlates of physical health of informal caregivers: a metaanalysis. Journals of Gerontology. 2007; 62:126-137. doi:10.1093/geronb/62.2.P126

34. National Institute for Clinical Excellence. Dementia: The NICE-SCIE guideline on supporting people with dementia and their careers in health and social care. London: The British Psychological Society and the Royal College of Psychiatrists; 2006.

35. Vérez Cotelo N, Andrés Rodríguez NF, Fornos Pérez JA, Andrés Iglesias JC, Mera Gallego R, Ríos Lago M. [Internet] Uso de psicótropos, plantas medicinales y vitaminas en cuidadores familiares de pacientes con Alzheimer. Farmacéuticos Comunitarios 2016; 8(Supl. 1). [Acceso 18/6/2016]. Disponible en: http://farmaceuticoscomunitarios.org/es/journal-article/ comparacion-psicopatologias-apoyo-prestado-cuidadores-enfermos-alzheimer-farmacia 